\title{
Increased ski expression levels are associated with a higher risk and poor prognosis in patients with gastrointestinal stromal tumors
}

\author{
QIU-SHI WANG ${ }^{1-3}$, YU ZHAI ${ }^{1},{\text { PING } \text { LI }^{1}, \text { HUA-LIANG XIAO }}^{2}$ and YUAN-GUO ZHOU ${ }^{1}$ \\ ${ }^{1}$ Molecular Biology Center, State Key Laboratory of Trauma, Burn and Combined Injury, Research Institute of Surgery \\ and Daping Hospital, The Army Medical University; ${ }^{2}$ Department of Pathology; ${ }^{3}$ Clinical Biobank, Daping Hospital \\ and Research Institute of Surgery, The Army Medical University, Chongqing 400042, P.R. China
}

Received June 10, 2019; Accepted November 21, 2019

DOI: $10.3892 / \mathrm{ol} .2020 .11280$

\begin{abstract}
Gastrointestinal stromal tumors (GISTs) are the most commonly diagnosed primary mesenchymal tumors of the gastrointestinal tract and $30 \%$ of GISTs are associated with a high recurrence risk or metastasis. The current risk classification criteria of the National Comprehensive Cancer Network are based on tumor size, mitotic activity and localization. Investigating additional biomarkers associated with clinical risk may aid in the diagnosis of GIST and improves prediction of patient prognosis. In the present study, the value of using the expression levels of the oncoprotein ski as a prognostic predictor for GISTs was investigated. The results demonstrated that high ski expression levels were correlated with high risk and recurrence rates and indicated poor prognosis regarding median disease-free survival. Overall, the present study suggests that ski expression levels may serve as a predictor for clinical risk and prognosis of patients with GISTs.
\end{abstract}

\section{Introduction}

Gastrointestinal stromal tumors (GISTs) are the most commonly reported primary mesenchymal tumors of the gastrointestinal tract (1) and 30\% of GISTs are highly malignant, with a high risk of abdominal and liver metastases (2). Therefore, it is important to improve the risk prediction for patients with a GIST prior to clinical treatment, to aid in the identification of patients who may require adjuvant imatinib therapy post-surgery (3). Patients with tumors classified as

Correspondence to: Professor Yuan-Guo Zhou, Molecular Biology Center, State Key Laboratory of Trauma, Burn and Combined Injury, Research Institute of Surgery and Daping Hospital, The Army Medical University, 10 Changjiang Zhilu Road, Chongqing 400042, P.R. China

E-mail:ygzhou@tmmu.edu.cn

Key words: ski, gastrointestinal stromal tumors, risk classification, prognosis high risk have a poor prognosis and a high risk of recurrence and metastasis (4). The current risk classification criteria of the National Comprehensive Cancer Network (NCCN) are based on tumor size, mitotic activity and primary lesion localization, whereas tumor site and gastrointestinal bleeding are independent risk factors of a poor prognosis for patients with GISTs $(5,6)$. Identifying biomarkers associated with clinical risk may aid diagnosis and improve the prognostic prediction of patients with GISTs.

As an auxiliary transcriptional regulator, ski is involved in nervous system development (7), proliferation and differentiation of hematopoietic cells (8), muscle differentiation (9), regeneration and wound healing (10). Furthermore, ski is overexpressed in a number of different types of cancer, including colorectal, gastric, pancreatic and lung cancer (11-14). Our previous study demonstrated that high ski expression levels in fibrosarcoma were associated with poor tumor cell differentiation and a less favorable prognosis, and that ski significantly promoted fibrosarcoma cell proliferation (data not yet published). Fibrosarcoma and GISTs are both primary mesenchymal tumors, therefore the potential value of ski expression levels as a prognostic biomarker and risk classifier in GISTs was investigated in the present study.

\section{Materials and methods}

Patient and tissue samples. The present study was approved by The Clinical Ethics Committee of Daping Hospital and Research Institute of Surgery, Army Medical University (Chongqing, China). Cases newly diagnosed as GIST by pathologists from January 2013 to December 2014 were included. Patients whose specimens did not qualify for immunohistochemistry, had missing clinical data or contact information were excluded. Patients provided informed written consent and were informed that their clinical data may be used in publications. A total of 81 tissue samples were obtained from patients diagnosed with pathologically verified GISTs from the Department of Gastrointestinal Surgery of Daping Hospital (Chongqing, China), including 38 samples from male patients and 43 samples from female patients (mean age, 61 years; age range, 35-80 years). Patient 
clinical and pathological data were obtained from patient medical records. Follow-up was performed by telephone every 2 months, starting from the date of primary diagnosis after the surgery and ending at any observable relapse (local recurrence and/or distant metastasis).

Immunohistochemistry (IHC). Rabbit polyclonal ski antibody (cat. no. sc-9140; Santa Cruz Biotechnology, Inc.) was used to determine ski expression levels. Positive and negative fibrosarcoma samples from our previous study (data not yet published) were used as positive and negative controls. Formalin-fixed and paraffin-embedded (4\% neutral formalin for $6-12 \mathrm{~h}$ at room temperature) tissues were sliced into $4-\mu \mathrm{m}$-thick sections. The tissues were subsequently deparaffinized and pretreated with $1 \mathrm{mmol} / \mathrm{LDTA}$ at $\mathrm{pH} 9.0$ in a high-pressure cooker for $3 \mathrm{~min}$ and then treated with $3 \%$ $\mathrm{H}_{2} \mathrm{O}_{2}$ for $10 \mathrm{~min}$. Subsequently, washing was performed for 3 min three times using $0.01 \mathrm{M}$ PBS at room temperature, and the slides were incubated with an anti-ski antibody (1:500) in the humidified chamber at $4^{\circ} \mathrm{C}$ overnight. Slides were washed the next day and incubated with 3,3'-diaminobenzidine stain for $5 \mathrm{~min}$ at room temperature, viewed under a bright-field microscope (BX41; Olympus) and images were captured by Cell Sens Standard software (version 1.16; Olympus) and analyzed blinded by two pathologists. Ski staining was scored as: Strong staining, 3+; moderate staining, 2+; faint staining, $1+$; or absence of staining, 0 .

Statistical analysis. All analyses were performed using SPSS software (version 18.0; SPSS Inc.). The correlation between ski expression levels with sex, age, tumor location and clinical risk classification were determined using a two-tailed t-test, Pearson's correlation analysis with Bonferroni's correction, ANOVA with a post-hoc Tukey's test and Spearman's correlation analysis with Bonferroni's correction, respectively. The Kaplan-Meier method was used to assess the prognostic value of ski expression levels for patients with GIST. A log-rank test was used to compare disease-free survival (DFS) curves and a Cox proportional hazards model was used to calculate univariate hazard ratios for the variables. $\mathrm{P}<0.05$ was considered to indicate a statistically significant difference.

\section{Results}

Patients' characteristics. Primary GIST tissue samples from 81 patients were analyzed. Univariate survival analysis showed that sex and age were not associated with patient prognosis ( $\mathrm{P}=0.06$ and $\mathrm{P}=0.8$, respectively). Tumors were localized in: Stomach, 69\%; small intestine, 16\%; esophagus, $4 \%$; enterocoelia, $7 \%$; pelvis, $3 \%$; and liver, $1 \%$. Detailed clinicopathological characteristics of the patients are presented in Table I.

Ski expression levels are correlated with clinical risk classification. The NCCN risk classification standard was used for GIST clinical classification. Patients were divided into four risk groups: High risk, 29 cases; medium risk, 17 cases; low risk, 21 cases; and extremely low risk, 14 cases. Univariate survival analysis demonstrated that classification as extremely low risk was significantly correlated with longer DFS ( $\mathrm{P}=0.009$; Table I). Patients were also divided into four groups based on IHC ski expression levels: $3+, 20$ cases; $2+, 24$ cases; $1+, 18$ cases; and $0+, 19$ cases; (Fig. 1). The numbers of patients with different levels of ski expression and in each clinical risk classification are shown in Table II. Higher ski expression levels were not significantly correlated with higher GIST clinical risk and sex, age and tumor location were not correlated with ski expression levels (Fig. 2)

Higher ski expression levels are correlated with higher recurrence risk of GISTS. Follow-up data from 71 patients with GISTs were collected to determine the correlation between recurrence rates and DFS with ski expression levels. These data were analyzed using the Kaplan-Meier method. Fewer patients with low ski expression levels (IHC 0 and IHC +1 ) had tumor recurrence at 36 months compared with patients with high ski expression levels (IHC $2+$ and 3+; 21 vs. 32\%, respectively). At the 80-month follow-up period, a larger number of patients with high ski expression levels had tumor recurrence compared with patients with low ski expression levels (55.3 vs. $24.2 \%$ respectively; Table III). Kaplan-Meier curves showed that the median DFS for the high ski expression group was shorter compared with the low ski expression group (72 months vs. N/A, respectively); however, this difference was not significant ( $\mathrm{P}=0.0586$; Fig. 3).

\section{Discussion}

Tumor size and mitotic index are the two primary factors used to assess tumor risk when utilizing the risk stratification system of the National Institutes of Health consensus (15). However, tumor location has subsequently been shown to have independent prognostic value, therefore, this factor was added into the risk stratification system and named the Miettinen-Lasota/Armed Forces Institute of Pathology classification system (16). Tumor size, location and mitotic index are the primary pathological factors of the risk classification system recommended by the European Society of Medical Oncology and NCCN, and are currently used in GIST guidelines (16). Additional prognostic factors have been reported for GIST, for example, activation of stem cell factor receptor (KIT) is involved in tumorigenesis of GISTs and specific KIT mutation types and loci have demonstrated prognostic value (17). Deletions in exon 11 of the KIT gene, particularly those involving codons 557 and/or 558, are associated with higher risk of metastases and have been reported as independent adverse prognostic factors $(18,19)$. Liu et al (6) reported that gastrointestinal bleeding was an independent risk factor for recurrence. Furthermore, the Japanese Kinki GIST registry group showed that pre or perioperative 'GIST rupture' was associated with a shorter median overall survival compared with patients with GISTs without 'tumor rupture' (6.4 vs. 11.9 years, respectively) (20). However, the clinical definition of 'tumor rupture' is impractical and ambiguous, therefore it is difficult to use 'tumor rupture' as an independent prognostic factor for GIST (21).

High ski expression levels are associated with poor prognosis in a number of types of cancer, such as esophageal carcinoma $(22,23)$, leukemia $(24)$, pancreatic cancer $(25,26)$ and melanoma (27). However, to the best of our knowledge, the 
Table I. Univariate analysis of disease-free survival of the 81 patients with gastrointestinal stromal tumors.

\begin{tabular}{|c|c|c|c|c|}
\hline Characteristic & No. patients $(\%)$ & HR & $95 \% \mathrm{CI}$ & P-value \\
\hline Sex & & 2.21 & $1.02-5.07$ & 0.060 \\
\hline Male & $38(47)$ & & & \\
\hline Female & $43(53)$ & & & \\
\hline Age & & 0.91 & $0.40-2.01$ & 0.800 \\
\hline$<60$ years & $37(46)$ & & & \\
\hline$\geq 60$ years & $44(54)$ & & & \\
\hline \multicolumn{5}{|l|}{ Location } \\
\hline Gastric & $56(59)$ & 1.94 & $0.65-5.76$ & $0.240^{\mathrm{a}}$ \\
\hline Small intestinal & $13(16)$ & & & \\
\hline Esophagus & $3(4)$ & 4.04 & $1.62-10.09$ & $0.003^{b}$ \\
\hline Enterocoelia & $6(7)$ & & & \\
\hline Pelvic & $2(3)$ & & & \\
\hline Liver & $1(1)$ & & & \\
\hline NCCN risk classification & & 0.33 & $0.17-0.75$ & 0.009 \\
\hline Very low & $14(17)$ & & & \\
\hline Low & $21(26)$ & & & \\
\hline Moderate & $17(21.0)$ & & & \\
\hline High & $29(36)$ & & & \\
\hline
\end{tabular}

HR, hazard ratio; CI, confidence interval; NCCN, National Comprehensive Cancer Network. ${ }^{\mathrm{a}} \mathrm{vs}$. gastric; ${ }^{\mathrm{b}}$ locations of esophagus, enterocoelia, pelvic and liver were analyzed as a group versus gastric.

Table II. Ski expression levels in IHC and risk classification of patients with gastrointestinal stromal tumors.

\begin{tabular}{|c|c|c|c|c|c|}
\hline NCCN risk classification & $0+$ & $1+$ & $2+$ & $3+$ & Total no. of patients \\
\hline Very low & 8 & 5 & 0 & 1 & 14 \\
\hline Low & 5 & 3 & 9 & 4 & 21 \\
\hline Moderate & 2 & 5 & 6 & 4 & 17 \\
\hline High & 4 & 5 & 9 & 11 & 29 \\
\hline Total no. of patients & 19 & 18 & 24 & 20 & 81 \\
\hline
\end{tabular}

NCCN, National Comprehensive Cancer Network; IHC, immunohistochemistry; 0+, absence of staining; 1+, faint staining; 2+, moderate staining; $3+$, strong staining.

present study is the first to demonstrate the correlations between ski expression levels and its prognostic value for GISTs. High ski expression levels were correlated with a high clinical risk of GISTs, but not correlated with sex, age or tumor location. Together, these data suggest that ski expression levels are correlated with the prognosis of patients with GIST. Follow-up data showed that patients with GISTs in the high ski expression group had higher recurrence rates compared with patients in the low ski expression group. These data suggest that patients with GISTs with high ski expression levels may have a higher risk of recurrence during the first 3 years after complete surgical resection and diagnosis of a primary localized GIST. High ski expression levels were found to be a poor prognostic factor of median DFS; however, this difference was not significant which may be due to the small patient cohort size. Therefore, the results of the present study need to be validated using larger patient
Table III. Tumor recurrence rates for patients with gastrointestinal stromal tumors with low and high ski expression levels based on IHC.

\begin{tabular}{lcr}
\hline Recurrence status $(\%)$ & Low & \multicolumn{1}{c}{ High } \\
\hline Before 36 months & $7(21.2)$ & $12(31.6)$ \\
37 to 80 months & $1(3.0)$ & $9(23.7)$ \\
Recurrence free & $25(75.8)$ & $17(45.7)$ \\
\hline
\end{tabular}

IHC, immunohistochemistry; low, IHC score of +0 or +1 ; high, IHC score of +2 or +3 .

cohorts before ski expression levels can be used clinically as an independent biomarker of prognosis for patients with GIST. 


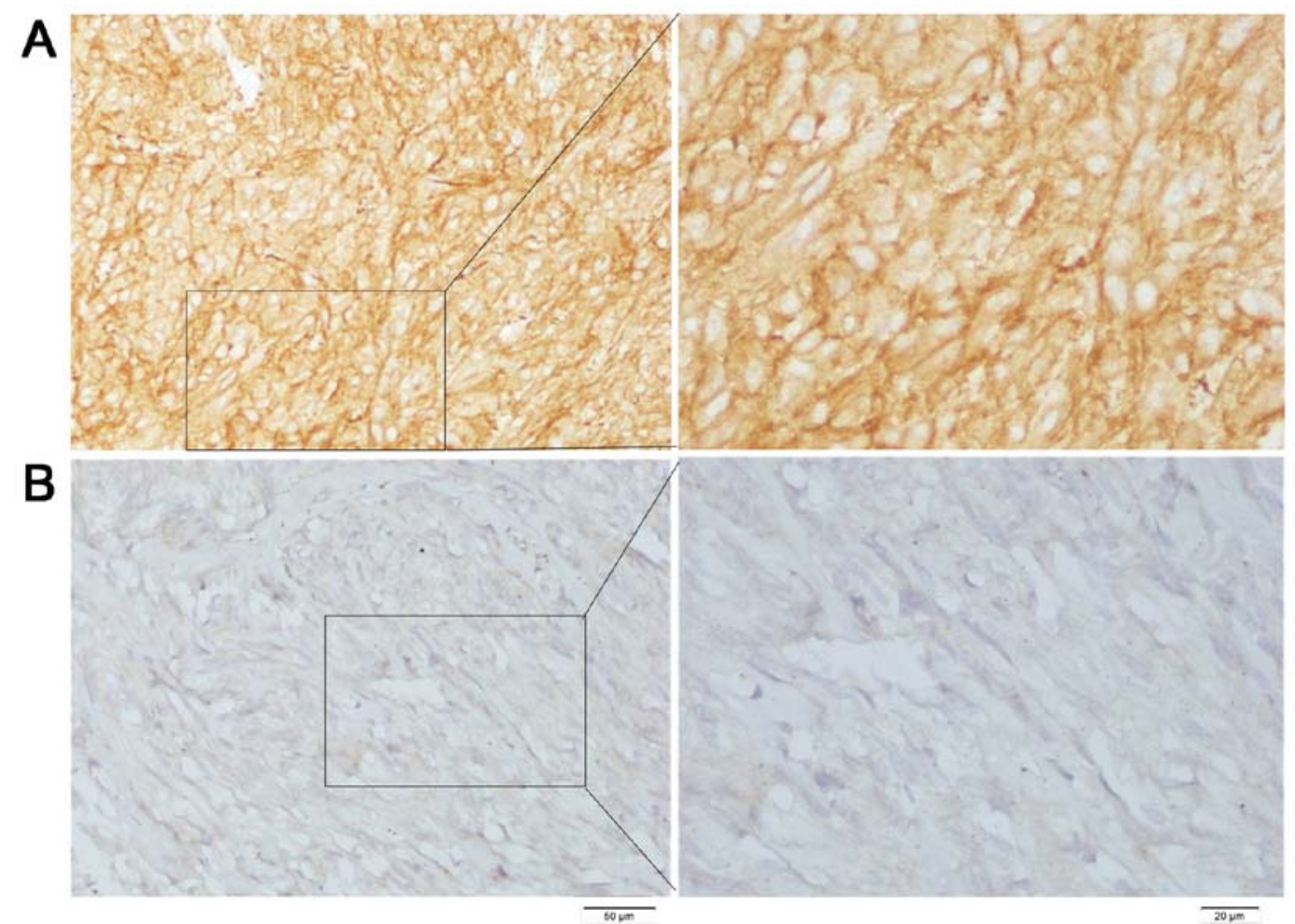

Figure 1. Ski expression levels in formalin-fixed and paraffin embedded tissues from patients with gastrointestinal stromal tumors. (A) Patient with IHC $3+$ ski expression levels. (B) Patient with IHC 0+ ski expression levels. IHC, immunohistochemical staining.
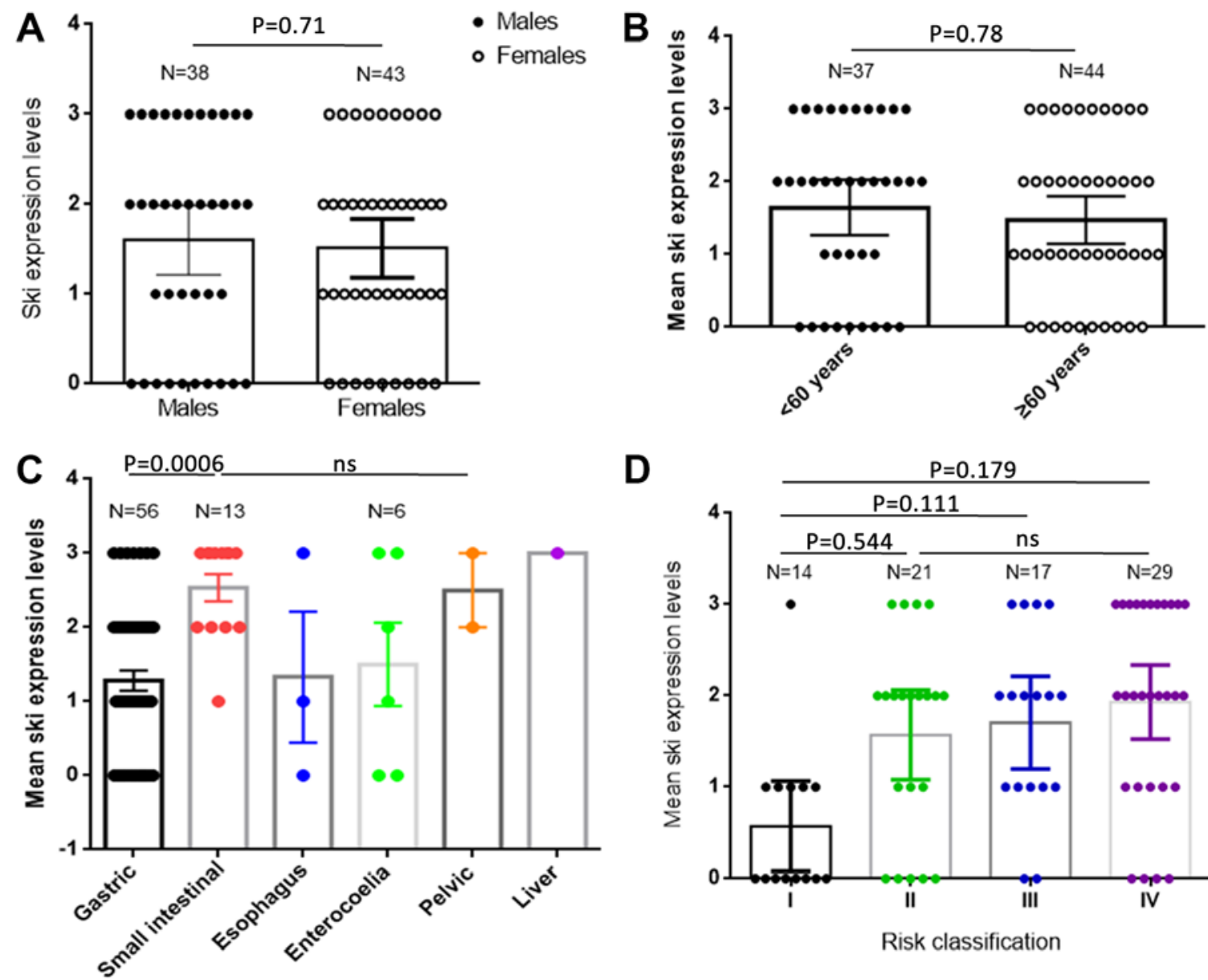

D

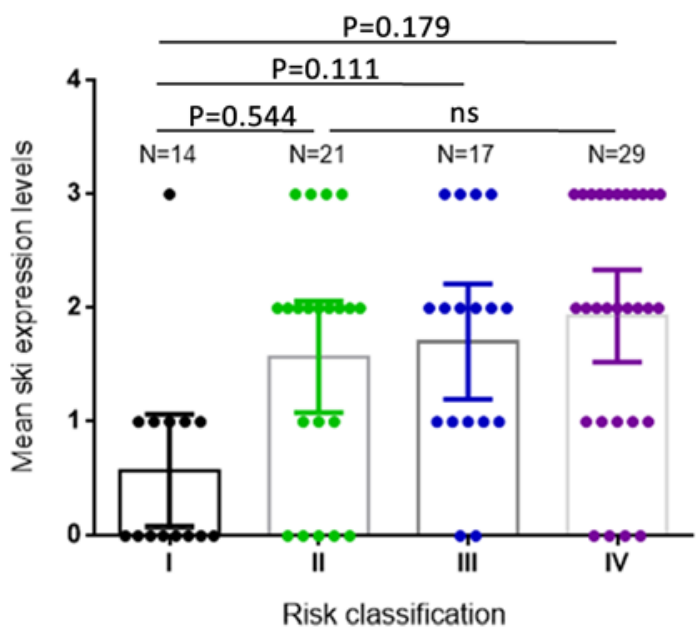

Figure 2. Correlation analysis of ski expression levels with clinicopathological characteristics. Correlation between ski expression levels and (A) sex, (B) age, (C) tumor location or (D) clinical risk classification. ns, no significant correlations. 
Ski higher expression indicated poor DFS

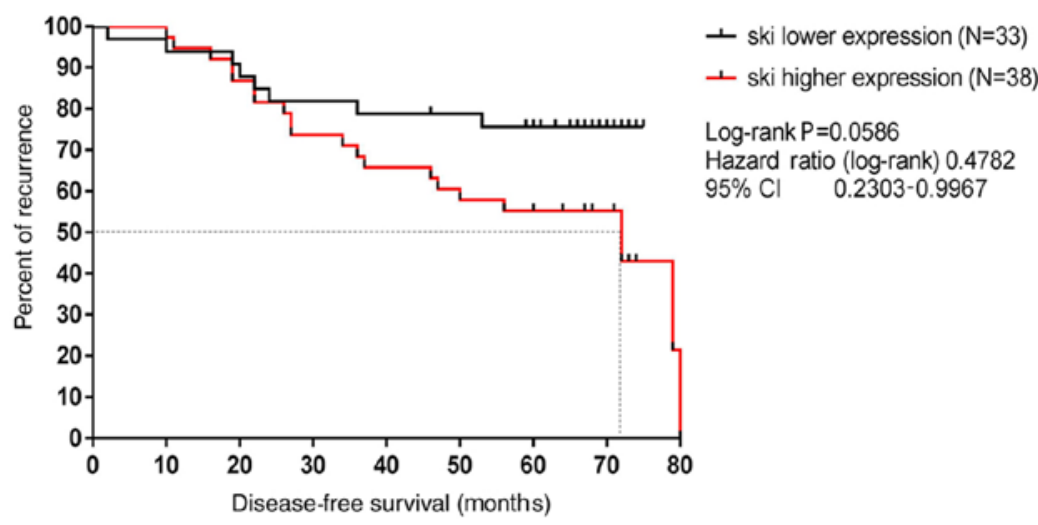

Figure 3. Association between ski expression levels and median DFS. Lower ski expression levels refers to an immunohistochemical staining score of +0 or +1 and higher ski expression levels refers to an immunohistochemical staining score of +2 or +3 . Patients with high ski expression levels had a lower median DFS compared with patients with low ski expression levels. DFS, disease-free survival; CI, confidence interval.

\section{Acknowledgements}

The authors would like to thank Mrs. Li Lin and Mrs. Ping Fu (Department of Pathology, Daping Hospital and Research Institute of Surgery, Army Medical University; Chongqing, China) for their assistance with immunohistochemistry, and Mr. He Xiao (Cancer center, Daping Hospital and Research Institute of Surgery, Army Medical University; Chongqing, China) for the assistance with statistical analysis

\section{Funding}

This work was supported by The National Natural Science Foundation of China (grant no. 81802781).

\section{Availability of data and materials}

The datasets used and/or analyzed during the present study are available from the corresponding author on reasonable request.

\section{Authors' contributions}

QSW, YGZ and HLX analyzed and interpreted the data. YZ and PL performed the experiments. QSW and YGZ wrote the manuscript. All the authors read and approved the final manuscript.

\section{Ethics approval and consent to participate}

The present study was approved by the Clinical Ethics Committee of Daping Hospital and Research Institute of Surgery, Army Medical University (Chongqing, China). Patients provided informed written consent and were informed that their clinical data may be used in publications.

\section{Patient consent for publication}

Not applicable.

\section{Competing interests}

The authors declare that they have no competing interests.

\section{References}

1. Du CY, Shi YQ, Zhou Y, Fu H and Zhao G: The analysis of status and clinical implication of KIT and PDGFRA mutations in gastrointestinal stromal tumor (GIST). J Surg Oncol 98: 175-178, 2008.

2. Mavroeidis L, Metaxa-Mariatou V, Papoudou-Bai A, Lampraki AM, Kostadima L, Tsinokou I, Zarkavelis G, Papadaki A, Petrakis D, Gkoura S, et al: Comprehensive molecular screening by next generation sequencing reveals a distinctive mutational profile of KIT/PDGFRA genes and novel genomic alterations: Results from a 20-year cohort of patients with GIST from north-western Greece. ESMO Open 3: e000335, 2018.

3. Lopes LF and Bacchi CE: Imatinib treatment for gastrointestinal stromal tumour (GIST). J Cell Mol Med 14: 42-50, 2010.

4. Sanchez Hidalgo JM, Rufian Peña S, Ciria Bru R, Naranjo Torres A, Muñoz Casares C, Ruiz Rabelo J and Briceño Delgado J: Gastrointestinal stromal tumors (GIST): A prospective evaluation of risk factors and prognostic scores. J Gastrointest Cancer 41: 27-37, 2010.

5. Liu X, Qiu H, Wu Z, Zhang P, Feng X, Chen T, Li Y, Tao K, Li G, Sun X, et al: A Novel pathological prognostic score (PPS) to identify 'very high-risk' patients: A multicenter retrospective analysis of 506 patients with high risk gastrointestinal stromal tumor (GIST). J Gastrointest Surg 22: 2150-2157, 2018.

6. Liu Q, Li Y, Dong M, Kong F and Dong Q: Gastrointestinal bleeding is an independent risk factor for poor prognosis in GIST patients. Biomed Res Int 2017: 7152406, 2017.

7. Stegmüller J, Konishi Y, Huynh MA, Yuan Z, Dibacco S and Bonni A: Cell-intrinsic regulation of axonal morphogenesis by the Cdh1-APC target SnoN. Neuron 50: 389-400, 2006.

8. Pearson-White S, Deacon D, Crittenden R, Brady G, Iscove N and Quesenberry PJ: The ski/sno protooncogene family in hematopoietic development. Blood 86: 2146-2155, 1995.

9. Sutrave P, Kelly AM and Hughes SH: Ski can cause selective growth of skeletal muscle in transgenic mice. Genes Dev 4: 1462-1472, 1990.

10. Liu X, Zhang E, Li P, Liu J, Zhou P, Gu DY, Chen X, Cheng T and Zhou Y: Expression and possible mechanism of c-ski, a novel tissue repair-related gene during normal and radiation-impaired wound healing. Wound Repair Regen 14: 162-171, 2006.

11. Bravou V, Antonacopoulou A, Papadaki H, Floratou K, Stavropoulos M, Episkopou V, Petropoulou C and Kalofonos H: TGF-beta repressors SnoN and Ski are implicated in human colorectal carcinogenesis. Cell Oncol 31: 41-51, 2009.

12. Kiyono K, Suzuki HI, Morishita Y, Komuro A, Iwata C, Yashiro M, Hirakawa K, Kano MR and Miyazono K: c-Ski overexpression promotes tumor growth and angiogenesis through inhibition of transforming growth factor-beta signaling in diffuse-type gastric carcinoma. Cancer Sci 100: 1809-1816, 2009.

13. Song L, Chen X, Gao S, Zhang C, Qu C, Wang P and Liu L: Ski modulate the characteristics of pancreatic cancer stem cells via regulating sonic hedgehog signaling pathway. Tumour Biol: Oct 12, 2016 (Epub ahead of print).

14. Yang H, Zhan L, Yang T, Wang L, Li C, Zhao J, Lei Z, Li X and Zhang HT: Ski prevents TGF- $\beta$-induced EMT and cell invasion by repressing SMAD-dependent signaling in non-small cell lung cancer. Oncol Rep 34: 87-94, 2015. 
15. Fletcher CD, Berman JJ, Corless C, Gorstein F, Lasota J, Longley BJ, Miettinen M, O'Leary TJ, Remotti H, Rubin BP, et al: Diagnosis of gastrointestinal stromal tumors: A consensus approach. Hum Pathol 33: 459-465, 2002.

16. Patel S: Navigating risk stratification systems for the management of patients with GIST. Ann Surg Oncol 18: 1698-1704, 2011.

17. Wozniak A, Rutkowski P, Piskorz A, Ciwoniuk M, Osuch C, Bylina E, Sygut J, Chosia M, Rys J, Urbanczyk K, et al: Prognostic value of KIT/PDGFRA mutations in gastrointestinal stromal tumours (GIST): Polish Clinical GIST Registry experience. Ann Oncol 23: 353-360, 2012.

18. Wardelmann E, Losen I, Hans V, Neidt I, Speidel N, Bierhoff E, Heinicke T, Pietsch T, Büttner R and Merkelbach-Bruse S: Deletion of Trp-557 and Lys-558 in the juxtamembrane domain of the c-kit protooncogene is associated with metastatic behavior of gastrointestinal stromal tumors. Int J Cancer 106: 887-895, 2003.

19. Martín J, Poveda A, Llombart-Bosch A, Ramos R, López-Guerrero JA, García del Muro J, Maurel J, Calabuig S, Gutierrez A, González de Sande JL, et al: Deletions affecting codons 557-558 of the c-KIT gene indicate a poor prognosis in patients with completely resected gastrointestinal stromal tumors: A study by the Spanish Group for Sarcoma Research (GEIS). J Clin Oncol 23: 6190-6198, 2005.

20. Nishida T, Cho H, Hirota S, Masuzawa T, Chiguchi G and Tsujinaka T; Kinki GIST Study Group: Clinicopathological features and prognosis of primary GISTs with tumor rupture in the real world. Ann Surg Oncol 25: 1961-1969, 2018.

21. Asare EA and Feig BW: Raining Frogs, flying horses, and defining tumor rupture in GIST. Ann Surg Oncol 26: 1601-1603, 2019.
22. Fukuchi M, Nakajima M, Fukai Y, Miyazaki T, Masuda N, Sohda M, Manda R, Tsukada K, Kato $\mathrm{H}$ and Kuwano H: Increased expression of c-Ski as a co-repressor in transforming growth factor-beta signaling correlates with progression of esophageal squamous cell carcinoma. Int J Cancer 108: 818-824, 2004.

23. Villanacci V, Bellone G, Battaglia E, Rossi E, Carbone A, Prati A, Verna C, Niola P, Morelli A, Grassini M and Bassotti G: Ski/SnoN expression in the sequence metaplasia-dysplasia-adenocarcinoma of Barrett's esophagus. Hum Pathol 39: 403-409, 2008.

24. Ritter M, Kattmann D, Teichler S, Hartmann O, Samuelsson MK, Burchert A, Bach JP, Kim TD, Berwanger B, Thiede C, et al: Inhibition of retinoic acid receptor signaling by Ski in acute myeloid leukemia. Leukemia 20: 437-443, 2006.

25. Heider TR, Lyman S, Schoonhoven R and Behrns KE: Ski promotes tumor growth through abrogation of transforming growth factor-beta signaling in pancreatic cancer. Ann Surg 246: 61-68, 2007.

26. Wang P, Chen Z, Meng ZQ, Fan J, Luo JM, Liang W, Lin JH, Zhou ZH, Chen H, Wang K, et al: Dual role of Ski in pancreatic cancer cells: Tumor-promoting versus metastasis-suppressive function. Carcinogenesis 30: 1497-1506, 2009.

27. Boone $B$, Haspeslagh $M$ and Brochez L: Clinical significance of the expression of c-Ski and SnoN, possible mediators in TGF-beta resistance, in primary cutaneous melanoma. J Dermatol Sci 53: 26-33, 2009.

(1)(9) This work is licensed under a Creative Commons Attribution-NonCommercial-NoDerivatives 4.0 International (CC BY-NC-ND 4.0) License. 